\title{
The origin of the high work function of chlorinated indium tin oxide
}

\author{
Peng-Ru Huang ${ }^{1}$, Yao $\mathrm{He}^{1}$, Chao $\mathrm{Cao}^{2}$ and Zheng-Hong $\mathrm{Lu}^{1,3}$ \\ The impact of halogenation, in particular $\mathrm{Cl}$ and $\mathrm{F}$, on the work functions of indium tin oxide (ITO) surfaces was studied using \\ density functional theory calculations. We found that a strong surface dipole layer induced by the halogen, rather than a change \\ in the electrochemical potential (that is, Fermi level) of the ITO, led to a dramatic increase in the work function. However, the \\ work function for F-coated ITO was lower than that of Cl-coated ITO. This result contradicts the well-known fact that F is much \\ more electronegative than $\mathrm{Cl}$. Detailed computations reveal that both electronegativity and atomic size collectively contribute to \\ the extraordinarily high work function of $\mathrm{Cl}$-ITO. Additionally, the work function increases linearly with increasing surface \\ halogen coverage for both systems, which was consistent with experimental data.
}

NPG Asia Materials (2013) 5, e57; doi:10.1038/am.2013.33; published online 9 August 2013

Keywords: charge transfer; chlorination; indium tin oxide; work function

\section{INTRODUCTION}

The physical process involved in charge transfer at an electrodemolecule interface has a critical role in many electronic devices, such as dye-sensitized solar cells, ${ }^{1,2}$ organic photovoltaic cells, ${ }^{3}$ organic light-emitting diodes, ${ }^{4-6}$ photocatalytic converters ${ }^{7-9}$ and so on. The energy barrier at this interface dictates the functionality of all molecule-based devices. It was recently discovered that the surface work function of metal oxide electrodes is one of the most important parameters in determining the interfacial barrier. Owing to its unique combination of good electrical conductivity and excellent optical transmission properties, tin-doped indium oxide (ITO) has been well developed. ITO is now a deeply entrenched industrial electrode material that is widely used in devices ranging from liquid crystal displays and solar cells to organic light-emitting diodes. ${ }^{10-14}$ However, the surface work function of ITO $(\sim 4.7 \mathrm{eV})$ is too low for organic semiconductor devices, where the highest occupied molecular orbital of the hole transport host organic semiconductors is typically $\sim 6.0 \mathrm{eV}$. To compensate for this substantial energy difference, several additional organic semiconductor buffer layers with highest occupied molecular orbitals between 4.7 and $6.0 \mathrm{eV}$ are inserted into the devices. These additional layers not only cause increased complexity in device fabrication, but also lead to an increased polariton-exciton interaction. ${ }^{15,16}$

A recent breakthrough in boosting the ITO surface work function by surface chlorination has been reported by Helander et al. ${ }^{14}$ Chlorinated ITO (Cl-ITO) has become a promising electrode for many devices. ${ }^{17-23}$ After experimentally treating with o-dichlorobenzene, the work function of the Cl-ITO surface was found to increase from 4.7 to $6.1 \mathrm{eV}$, which directly correlates with the highest occupied molecular orbital of the hole transport organic semiconductors. This unprecedentedly high work function enables a much simpler device structure, yet yields a record current efficiency at high luminance. Following this work, methods such as chlorineplasma treatment, ${ }^{21} \mathrm{Cl}_{2}$-plasma etching, ${ }^{22}$ indium trichloride evaporation $^{23}$ and chloroform treatment ${ }^{20}$ were developed to achieve ITO chlorination. These techniques drastically modify the work function of ITO and enhance device performance. Despite the progress made in fabricating various types of devices based on $\mathrm{Cl}$ ITO, a thorough understanding of the unexpectedly high work function of chlorinated ITO is still needed. Previously, the large difference in the electronegativity of In and $\mathrm{Cl}$, which results in a surface dipole layer, was proposed to explain the high work function of chlorinated ITO. ${ }^{14,21,22}$ If electronegativity is the only driving force for work-function modification, ITO modified with more electronegative species, for example, fluorine, should result in a larger work function. However, a work function higher than that observed for chlorinated ITO has never been reported. Thus, in this study, we used first-principle calculations within density functional theory to understand the work-function modification by coating ITO surfaces with two different elements- $\mathrm{Cl}$ and $\mathrm{F}$.

\section{MATERIALS AND METHODS}

Our density functional theory calculations were performed using the Vienna $a b$ initio simulation package (VASP: Vienna, Austria) with the projectoraugmented wave pseudopotentials. ${ }^{24-27}$ We used the Perdew-Burke-Ernzerhof generalized gradient approximation to treat the exchange-correlation energy of interacting electrons. ${ }^{28}$ The energy cutoff for the plane-wave basis set was $500 \mathrm{eV}$. A $(4 \times 4 \times 1)$ Monkhorst-Pack $k$-point grid was used for the

${ }^{1}$ Department of Physics, Yunnan University, Kunming, China; ${ }^{2}$ Department of Physics, Hangzhou Normal University, Hangzhou, China and ${ }^{3}$ Department of Materials Science and Engineering, University of Toronto, Toronto, Ontario, Canada 
calculation of Cl- or F-coated ITO surfaces. All considered structures were fully optimized with the force on each atom being $<0.01 \mathrm{eV}^{-1}$.

The crystal structure of $\operatorname{In}_{2} \mathrm{O}_{3}$ is cubic bixbyite (space group: Ia $\overline{3}$, lattice constant: $10.117 \AA) .{ }^{29}$ Indium atoms occupy Wyckoff positions of $8 b$ and $24 d$, while oxygen atoms occupy Wyckoff positions of 48e. Our calculated equilibrium structure keeps the initial symmetry but has a slightly larger lattice constant of $10.30 \AA$, which is consistent with previous calculations using the same functional. ${ }^{30}$

As shown in Figure 1, we chose a slab with nine atomic layers separated by $20 \AA$ of vacuum to model the ITO surface. The slab was constructed by cleaving the bulk oxide along the (001) plane and substituting one-eighth of the In atoms with $\mathrm{Sn}$ atoms on the two surfaces. The surface with both $24 d$ and $8 b$ In atoms exposed was chosen in our calculations for its homogenous bond breaking. By varying the thickness of the slab and the width of the vacuum layer separating the periodic images perpendicular to the surface, we verified that the selected system size provided convergent results.

We studied the adsorption characteristics of $\mathrm{Cl}$ or F on an ideal ITO (001) $(1 \times 1)$ surface by placing the adatoms symmetrically on each side of the slab. Three types of adsorption sites were considered in our calculation: the top site above a metal atom, the bridge site between two adjacent metal atoms and the hollow site at the center of the quadrilateral grid ( $c f$., Figure 1b). As a real ITO (001) surface displays highly complex reconstructions and is computationally demanding, our discussions are constrained to $\mathrm{Cl}$ or F on an ideal ITO (001) $(1 \times 1)$ surface. Our main findings show that the work-function modification is localized and dependent on induced surface dipole, which is dictated by coordination and the geometry of the local bonding. From this perspective, our conclusions could be qualitatively extended to more complex surface structures.

The work function is the minimum energy needed to remove an electron from the bulk of a material through the surface to a point outside the material and is defined as

$$
\Phi=V_{\text {vac }}-E_{\mathrm{F}}
$$

where $E_{\mathrm{F}}$ is the Fermi level and $V_{\text {vac }}$ is the vacuum level, that is the potential energy of electrons outside the material. The calculated work function for bare
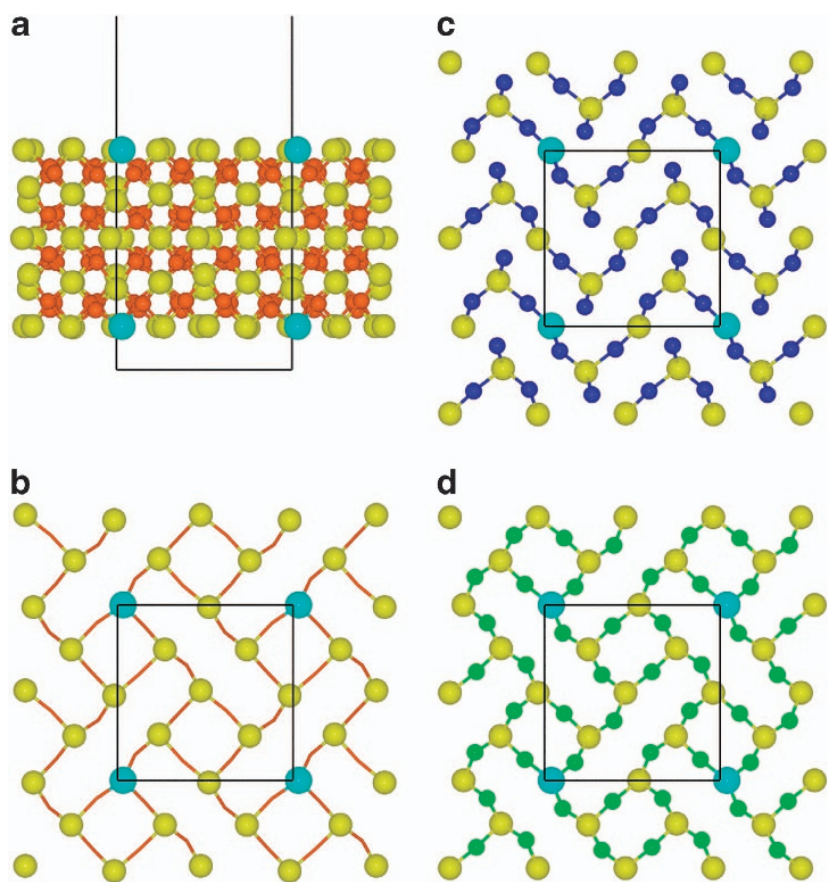

Figure 1 Side view (a) and top view (b) of metal-terminated ITO (001) surface. Top view of the adsorption structure of $\mathrm{Cl}$-coated (c) and $\mathrm{F}$-coated (d) ITO at surface coverage of $\theta=1.5$. Atomic species are color coded as follows: In (yellow), O (red), Sn (turquoise), $\mathrm{Cl}$ (blue), and $\mathrm{F}$ (green). Black lines indicate the unit cell edges.
ITO surfaces is $4.62 \mathrm{eV}$, which is close to the experimental value of $\sim 4.7 \mathrm{eV}$ for untreated ITO surfaces.

\section{RESULTS AND DISCUSSION}

We first consider the adsorption characteristics of $\mathrm{Cl}$ atoms on the (001) surface of ITO at different coverage levels. The surface coverage, $\theta$, is defined as the ratio between the number of adatoms and surface atoms. The stable adsorption geometries of $\mathrm{Cl}$ on ITO surfaces were identified by comparing the adsorption energy at different adsorption sites, which is defined as

$$
E_{\mathrm{ads}}(n)=\frac{1}{n}\left[E_{\mathrm{Cl}-\mathrm{ITO}}-E_{\mathrm{ITO}}-\frac{n}{2} E_{\mathrm{Cl}_{2}}\right]
$$

where $E_{\mathrm{Cl} \text {-ITO }}$ is the total energy of the Cl-coated ITO, $E_{\mathrm{ITO}}$ is the energy of the ITO substrate, $n$ is the number of $\mathrm{Cl}$ adatoms and $E_{\mathrm{Cl}_{2}}$ is the energy of a chlorine molecule. At a coverage of $\theta=0.125$, the most stable adsorption geometry is a $\mathrm{Cl}$ atom adsorbed on top of an In atom. The adsorption energy is $-1.85 \mathrm{eV} /$ per adatom, and the $\mathrm{Cl}$ In bond length is $2.37 \AA$. Other adsorption geometries are unstable because of their adsorption energies. The adsorption energies are $-1.60 \mathrm{eV}$ per adatom for the bridge site between two adjacent In atoms, $-0.60 \mathrm{eV}$ per adatom on top of $\mathrm{Sn}$ atoms and $-0.82 \mathrm{eV}$ per adatom for the hollow site at the center of the quadrilateral grid (Figure 1b). Our results indicate that $\mathrm{Cl}-\mathrm{Sn}$ bonding was unlikely given the higher adsorption energy $(\sim 1.25 \mathrm{eV}$ per adatom) on top of Sn compared to that of In. The high-resolution XPS spectra of the $\mathrm{Cl}$ $2 p$ core level on a chlorinated ITO surface have shown that $\mathrm{Cl}$ adatoms are chemically bonded to In atoms, ${ }^{14}$ which agrees with our calculations. When the surface coverage, $\theta$, is $>0.5$, the interactions between adjacent $\mathrm{Cl}$ adatoms become dominan0t, and the adsorption geometries are somewhat modified. $\mathrm{Cl}$ adatoms moved to the bridge site, which was only $0.25 \mathrm{eV}$ per adatom higher than the top site at the low coverage of $\theta=0.125$. The highest surface coverage, $\theta$, was 1.5 when surface dangling bonds of the ITO substrate were completely saturated. At this surface coverage, $\mathrm{Cl}$ adatoms formed a close packing pattern (Figure 1c) and displayed a mixing configuration of adsorption on top and bridge sites due to the stronger interactions between $\mathrm{Cl}$ adatoms. The adsorption energies were found to be $-1.33 \mathrm{eV}$ per adatom and $0.52 \mathrm{eV}$ per adatom higher than that of $\theta=0.125$.

To explore the work-function modification of an ITO surface upon $\mathrm{Cl}$ adsorption, we calculated the Fermi level, vacuum level and work function of a Cl-coated ITO (001) surface as a function of surface coverage, $\theta$. From the results plotted in Figure 2a, we determined that the position of the Fermi level $(-0.2 \pm 0.2 \mathrm{eV})$ remained independent of $\mathrm{Cl}$ coverage, $\theta$. This phenomenon is known as the Fermi-level pinning and is exhibited by many semiconductor surfaces in which the Fermi level is fixed at a particular level due to the existence of surface states that act either as donors or acceptors. This theoretical calculation is consistent with experimental data and shows no obvious change of electronic structure for chlorinated ITO. ${ }^{14}$ As the Fermi level was unaltered, the change in the work function of Cl-ITO was induced by a change in the vacuum level, as shown in Figure 2b.

Figure $2 \mathrm{a}$ shows that the vacuum level increased linearly with surface coverage, $\theta$. Therefore, according to equation 1 , there was also a near-linear increase of the work function, $\Phi$, as depicted in Figure 2a. The experimental work functions extracted from the study by Helander et al. ${ }^{14}$ were also plotted in Figure 2c. As shown, the theoretical results are in agreement with the experimental findings. The highest work function achieved during the experiments was $6.1 \mathrm{eV}$, which corresponds to our calculated value at a surface coverage of $\sim 1.0$. It is worth noting that our calculations also predict the 

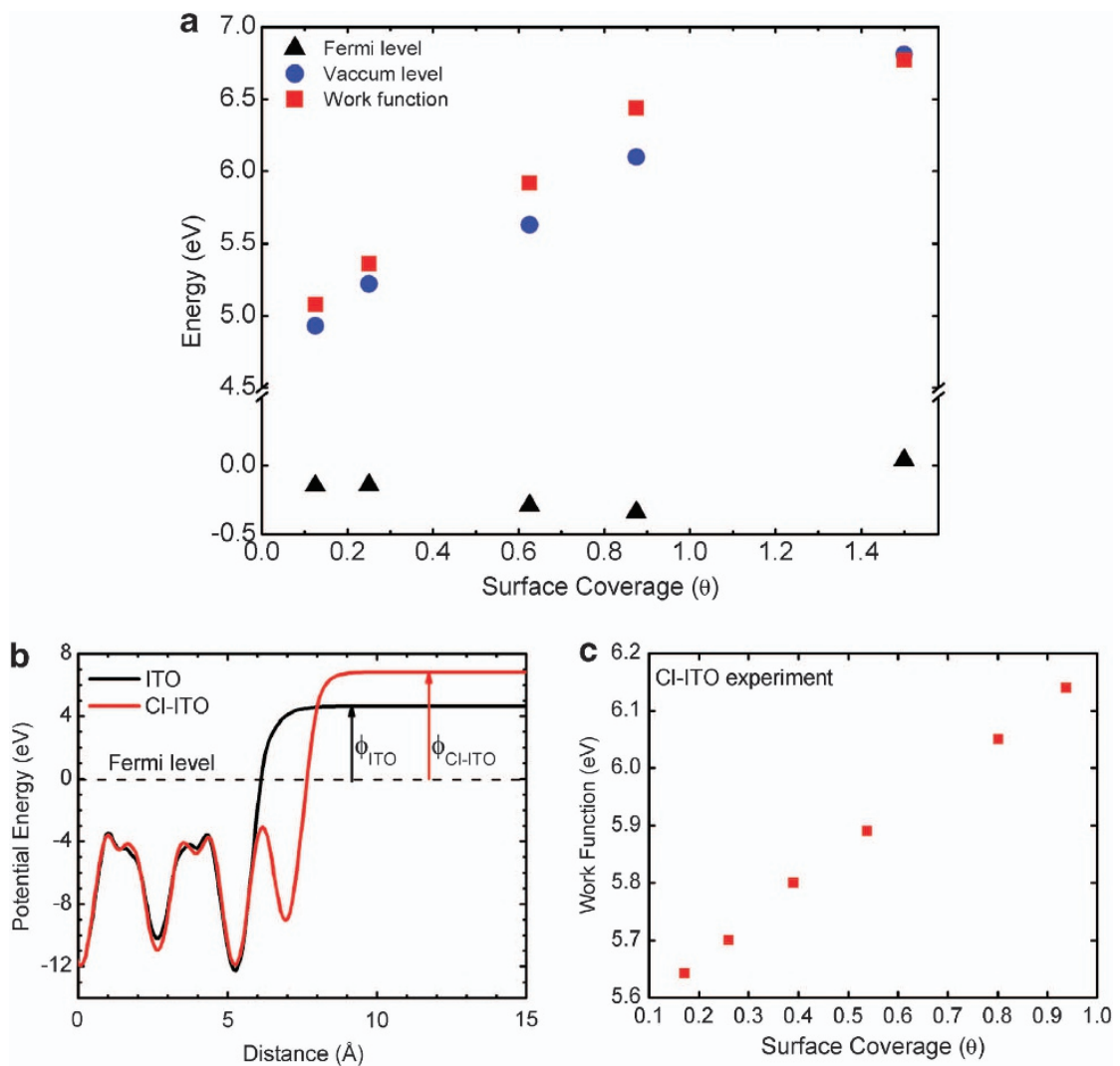

Figure 2 (a) Fermi level, vacuum level and the work function for Cl-coated ITO vs surface coverage, $\theta$. Black triangles, blue circles and red squares represent Fermi level, vacuum level and work function, respectively. The break divides the energy axis into $[-0.5,0.5]$ and $[4.5,7.0]$ intervals. (b) Planeaveraged electrostatic potential energy for ITO (black line) and CI-ITO (red line) (at $\theta=1.5$ ) vs the distance from the slab center. The slab center represents the zero position. The horizontal line represents the Fermi level. Arrows indicate the work function, $\Phi$, for ITO (black arrow) and Cl-ITO (red arrow). (c) The experimental work functions of $\mathrm{Cl}$-ITO were measured by Helander et al. ${ }^{14}$

highest value $(6.8 \mathrm{eV})$ of the work function when all surface dangling bonds were saturated by $\mathrm{Cl}$ adatoms. Consequently, if experiments further improve the surface coverage, additional increases in the work function could be anticipated.

The microscopic origin of work-function modification also needs to be addressed. It is natural to expect that the adsorption of $\mathrm{Cl}$ will prompt a redistribution of the electron density to accommodate the adatom and to minimize its impact. Thus, we plotted the planeaveraged charge density difference of $\mathrm{Cl}$-coated ITO at a coverage of $\theta=1.5$ in Figure 3 (the results for other coverage values are qualitatively the same), which is calculated using the expression

$$
\Delta \rho(z)=\rho_{\mathrm{Cl}-\mathrm{ITO}}(z)-\rho_{\mathrm{Cl}-\mathrm{ITO}}^{\mathrm{ITO}}(z)-\rho_{\mathrm{Cl}-\mathrm{ITO}}^{\mathrm{Cl}}(z)
$$

the three terms on the right-hand side of Equation 3 represent the plane-averaged charge density for the full Cl-ITO system $\left(\rho_{\text {Cl-ITO }}(z)\right)$, the bare ITO surface $\left(\rho_{\text {Cl-ITO }}^{\text {ITO }}(z)\right)$ and the $\mathrm{Cl}$ adatoms layer $\left(\rho_{\mathrm{Cl}-\mathrm{CTO}}^{\mathrm{Cl}}(z)\right)$. The charge densities were averaged in the planes parallel to the ITO surface. One immediately obvious consequence of Figure 3 was the substantial depletion of electrons from the ITO surface and a subsequent accumulation of electrons around $\mathrm{Cl}$ adatoms. This outcome was due to the highly electronegative $\mathrm{Cl}$ adatoms possessing a strong attraction for electrons. A net electron transfer occurred from the ITO surface to the $\mathrm{Cl}$ adatoms layer. In Figure 3, this charge rearrangement appears to have localized around the immediate bonding region between the $\mathrm{Cl}$ adatoms and the surface metal atoms. It is obvious from this result that a surface dipole layer was introduced to modify the electrostatic potential energy. The electrostatic potential energy, $V(z)$ relating to $\Delta \rho(z)$, can be evaluated by integrating the one-dimensional Poisson's equation

$$
\frac{d^{2} V(z)}{d z^{2}}=-\Delta \rho(z) / \varepsilon_{0}
$$

As shown in Figure 3, $V(z)$ converged to a value $\Delta V$ far away from the surface. This additional potential energy step, $\Delta V$, across the bonding region between $\mathrm{Cl}$ adatoms and surface $\mathrm{In} / \mathrm{Sn}$ atoms, pushed the vacuum level, $V_{\text {vac }}$, upward relative to the Fermi level, $E_{\mathrm{F}}$ The surface work function then increased accordingly. As shown in Figure $2 \mathrm{~b}$, the calculated work-function increase, $\Delta \Phi$, was $2.19 \mathrm{eV}$, which was approximately equal to the potential energy step, $\Delta V$ $(2.05 \mathrm{eV})$. These results clearly reveal that the major contribution to the work-function modification of $\mathrm{Cl}$-coated ITO originated from a surface dipole layer induced by charge rearrangement. This nearly linear coverage dependence of the work function can be attributed to the approximate linear dependence between the potential energy step and surface dipole

$$
\Delta V(z)=\frac{e \mu(z)}{\varepsilon_{0} A}
$$

with increasing coverage, the potential energy step, $\Delta V$, increased as the number of dipoles per unit area increased. Consequently, the work function increases almost linearly with increasing coverage. 


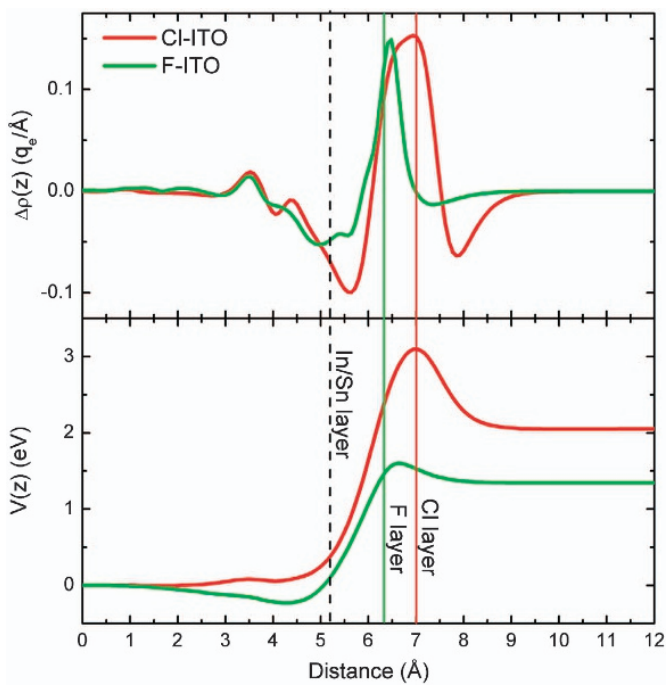

Figure 3 Plane-averaged charge density difference, $\Delta \rho(z)$, and potential energy change, $V(z)$, for $\mathrm{Cl}$-coated (red line) and F-coated (green line) ITO at surface coverage of $\theta=1.5$ vs the distance from the slab center. The slab center represents the zero position. The three vertical lines represent the position of the surface metal atom layer (dashed), $\mathrm{Cl}$ atom layer (red) and $\mathrm{F}$ atom layer (green).

At this point, the following questions arose: to what extent does the electronegativity of an element affect the work-function modification, and can we use highly electronegative elements to surpass the work function achieved by $\mathrm{Cl}$ ? Following the above microscopic description, one might conclude that the work function should be higher for an F-coated ITO surface, as fluorine is the most electronegative and reactive element and induces charge rearrangement more significantly. However, higher work function than chlorinated ITO has never been observed in experiments using highly electronegative atoms, such as F, $\mathrm{Br}$ and I. ${ }^{31-34}$ To decipher this contradiction, we investigated the work-function modification of the same ITO surface by adsorption of F, which was the most electronegative and reactive element. From an instructional perspective, the comparison and discussion of these two differently treated ITO substrates will help elucidate the role of different factors governing work-function modification.

The adsorption geometries and energies of F-coated ITO were investigated in the same manner as Cl-ITO. Our results showed that F adatoms always favored bridge sites and reappeared in the geometry of the bulk oxygen layer at a surface coverage of $\theta=1.5$ ( $c f$., Figure 1d). The adsorption energies of F-ITO varied only slightly (3.3-3.6 ev per adatom) with increasing coverage, which differed from those of Cl-ITO. This difference was likely due to the much smaller atomic size of $\mathrm{F}$, which led to less overlap of charge density between adatoms. Thus, the interactions between $\mathrm{F}$ adatoms had virtually no effect on the adsorption characteristics of $F$ on the ITO surface.

The adsorption of $\mathrm{F}$ on the ITO surface also resulted in a charge rearrangement resembling that on $\mathrm{Cl}-\mathrm{ITO}$, that is, the electron transfer occurred from the ITO surface to the F adatoms (cf., Figure 3). At the same time, a surface dipole layer and an additional potential energy step, $\Delta V$, built up across the bonding region between $\mathrm{F}$ adatoms and the surface metal atoms. Thus, the vacuum level shifted upward relative to Fermi level, and the work function increased almost linearly with increasing coverage ( $c f$., Figure 4). However, we note from Figure 4 that the slope of the work function

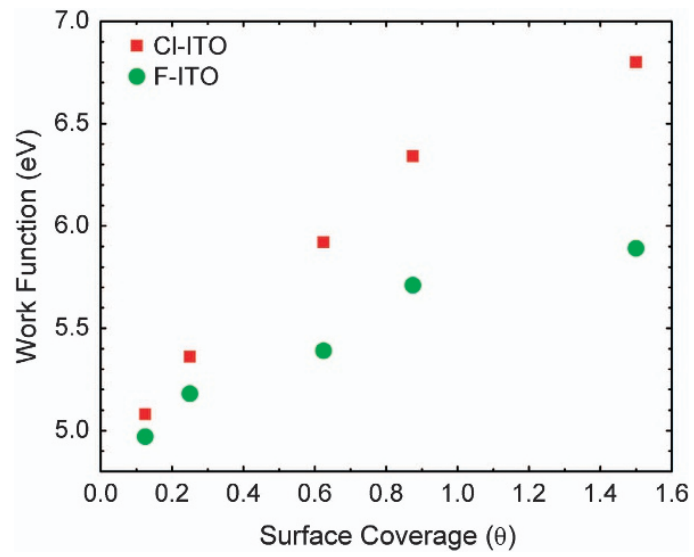

Figure 4 The calculated work function for $\mathrm{Cl}$-coated (red squares) and F-coated (green circles) ITO as a function of surface coverage, $\theta$.

Table 1 Potential energy step, $\Delta \boldsymbol{V}$; charge-transfer distance, $d$; and calculated charge transfer, $\Delta Q$ for $\mathrm{Cl}$ and F-coated ITO at surface coverage of $\theta=1.5$

\begin{tabular}{|c|c|c|c|c|c|}
\hline Adatom & $\begin{array}{l}\text { Potential } \\
\text { step (eV) }\end{array}$ & $\begin{array}{c}\text { Distance } \\
(A \circ)\end{array}$ & $\begin{array}{c}\text { Atomic } \\
\text { radius }(\AA)\end{array}$ & $\begin{array}{c}\text { Charge transfer } \\
\text { (e per atom) }\end{array}$ & Electronegativity \\
\hline $\mathrm{Cl}$ & 2.05 & 1.71 & 0.79 & 0.51 & 3.16 \\
\hline $\mathrm{F}$ & 1.34 & 1.07 & 0.42 & 0.71 & 3.98 \\
\hline
\end{tabular}

Atomic radius, $r$, and electronegativity (Pauling), $\chi$, for $\mathrm{Cl}$ and $\mathrm{F}$.

was not as steep as that seen with Cl-ITO, with the highest work function recorded at $5.8 \mathrm{eV}$, which was much less than that of Cl-ITO $(6.8 \mathrm{eV})$

This behavior was then analyzed using a simple electrostatic model. It is well known that dipole moment can be defined as the product of the magnitude of charge and the distance between the charges. In other words, two factors, the amount of charge transfer and the distance of charge transfer, determine the magnitude of the surface dipole on $\mathrm{Cl}$ - or F-coated ITO. The amount of charge transfer depends on the electronegativity difference between the adatom and the surface metal atom, while the distance is related to the bond length between them, that is, the size of the adatom and the surface metal atom. In Table 1, we present the atomic radius and electronegativity of $\mathrm{Cl}$ and $\mathrm{F}^{35}$ potential energy step, charge-transfer distance and calculated charge transfer with Bader analysis ${ }^{36}$ for $\mathrm{Cl}$ - and F-coated ITO surfaces. In this study, we approximated the chargetransfer distance by adatom-substrate spacing, $d$, which was calculated as the difference between the average $z$ coordinates of the adatoms and the surface metal atoms. The amount of charge transfer was 0.71 $e$ per adatom and $0.51 e$ per adatom from ITO to $\mathrm{F}$ and to $\mathrm{Cl}$, respectively. The charge transfer values for ITO to $\mathrm{F}$ were $\sim 28 \%$ greater than that of ITO to $\mathrm{Cl}$, as $\mathrm{F}$ was $\sim 21 \%$ more electronegative than $\mathrm{Cl}$. In the case of charge-transfer distance, the situation was completely opposite. The distance was $1.07 \AA$ for F-ITO, $\sim 60 \%$ smaller than the $1.71 \AA$ for Cl-ITO, which is consistent with the $\sim 88 \%$ smaller atomic radius of $\mathrm{F}$.

Based on the above discussion, the dominating factor in determining the surface dipole is clearly the charge-transfer distance, which is related to the bond length. According to equation 5, the potential energy step, $\Delta V$, is proportional to the magnitude of the surface dipole and will be higher for Cl-ITO. We obtained a $\sim 53 \%$ higher 
potential energy step, $\Delta V$, for Cl-ITO than for F-ITO (Table 1). A combination of the aspects described above was responsible for the work function being much higher for $\mathrm{Cl}$-coated ITO. Therefore, the combination of high electronegativity and large atomic size explains the extraordinarily high work function of Cl-ITO.

\section{Summary}

To summarize, we performed density functional theory calculations to investigate work-function modifications by coating ITO with $\mathrm{Cl}$ or $\mathrm{F}$. In particular, we studied and visualized the charge rearrangement and variation of electrostatic potential energy. Our results show that the work function increased linearly with increasing coverage for both systems. The same trends were also observed in the behavior of vacuum level and potential energy step. The surface dipole induced by charge rearrangement was responsible for the upward shift of vacuum level and the subsequent increase in the work function. However, the work-function increase for F-ITO was not as high as that for Cl-ITO; the highest work functions of F-ITO and Cl-ITO were 5.8 and $6.8 \mathrm{eV}$, respectively. Detailed theoretical analyses identified two major factors contributing to a drastic increase in the ITO work function: charge transfer to the halogen and adsorption bond length. As the atomic size was inversely proportional to the value of electronegativity, a balance between ionic radii and electronegativity is essential for reaching a high work function. The $\mathrm{Cl}$-coated ITO system reaches an optimal balance between the two competing factors, ultimately resulting in an extremely high work function. Furthermore, our calculations also predicted that the work function of $\mathrm{Cl}$-coated ITO could extend as high as $6.8 \mathrm{eV}$. The current analysis provides a theoretical framework for experimental efforts to engineer work functions of various material systems.

\section{CONFLICT OF INTEREST}

The authors declare no conflict of interest.

\section{ACKNOWLEDGEMENTS}

This work was supported by National Natural Science Foundation of China (Grant No. 11164032, 61066005 and U1037603); Chinese Ministry of Science and Technology (973 program, Grant No. 2012CB326400) and Program for New Century Excellent Talents in University (Grant No. NCET-12-1080). Computational resources were provided by the High Performance Computing Center of Yunnan University.

1 O'Regan, B. \& Grätzel, M. A low-cost, high-efficiency solar cell based on dye-sensitized colloidal $\mathrm{TiO}_{2}$ films. Nature 353, 737-740 (1991).

2 Gibson, E. A., Smeigh, A. L., Le Pleux, L., Fortage, J., Boschloo, G., Blart, E., Pellegrin, Y., Hagfeldt, A. \& Hammarström, L. A p-type NiO-based dye-sensitized solar cell with an open-circuit voltage of 0.35 V. Angew Chem. 121, 4466-4469 (2009).

3 Nattestad, A., Mozer, A. J., Fischer, M. K., Cheng, Y. B., Mishra, A., Bäuerle, P. \& Bach, U. Highly efficient photocathodes for dye-sensitized tandem solar cells. Nat. Mater. 9, 31-35 (2009).

4 Kabra, D., Lu, L. P., Song, M. H., Snaith, H. J. \& Friend, R. H. Efficient single-layer polymer light-emitting diodes. Adv. Mater. 22, 3194-3198 (2010).

5 Meyer, J., Khalandovsky, R., Görrn, P. \& Kahn, A. $\mathrm{MoO}_{3}$ films spin-coated from a nanoparticle suspension for efficient hole-injection in organic electronics. Adv. Mater. 23, 70-73 (2011)

6 Qiu, C., Xie, Z., Chen, H., Wong, M. \& Kwok, H. S. Comparative study of metal or oxide capped indium-tin oxide anodes for organic light-emitting diodes. J. Appl. Phys. 93, 3253-3258 (2003)

7 Roy, S. C., Varghese, O. K., Paulose, M. \& Grimes, C. A. Toward solar fuels: photocatalytic conversion of carbon dioxide to hydrocarbons. ACS Nano. 4, 1259-1278 (2010)

8 Chueh, W. C., Falter, C., Abbott, M., Scipio, D., Furler, P., Haile, S. M. \& Steinfeld, A. High-flux solar-driven thermochemical dissociation of $\mathrm{CO}_{2}$ and $\mathrm{H}_{2} \mathrm{O}$ using nonstoichiometric ceria. Science 330, 1797-1801 (2010).
9 Varghese, O. K., Paulose, M., LaTempa, T. J. \& Grimes, C. A. High-rate solar photocatalytic conversion of $\mathrm{CO}_{2}$ and water vapor to hydrocarbon fuels. Nano Lett. 9, 731-737 (2009)

10 Kido, J., Kimura, M. \& Nagai, K. Multilayer white light-emitting organic electroluminescent device. Science 267, 1332-1334 (1995).

11 Shen, Z., Burrows, P. E., Bulović, V., Forrest, S. R. \& Thompson, M. E. Three-color, tunable, organic light-emitting devices. Science 276, 2009-2011 (1997).

12 Friend, R. H., Gymer, R. W., Holmes, A. B., Burroughes, J. H., Marks, R. N., Taliani, C., Bradley, D. D. C., Dos Santos, D. A., Brédas, J. L., Lögdlund, M. \& Salaneck, W. R. Electroluminescence in conjugated polymers. Nature 397, 121-128 (1999).

13 Baldo, M. A., Thompson, M. E. \& Forrest, S. R. High-efficiency fluorescent organic light-emitting devices using a phosphorescent sensitizer. Nature 403, 750-753 (2000).

14 Helander, M. G., Wang, Z. B., Qiu, J., Greiner, M. T., Puzzo, D. P., Liu, Z. W. \& Lu, Z. H. Chlorinated indium tin oxide electrodes with high work function for organic device compatibility. Science 332, 944-947 (2011).

15 Aziz, H., Popovic, Z. D., Hu, N. X., Hor, A. M. \& Xu, G. Degradation mechanism of small molecule-based organic light-emitting devices. Science 283, 1900-1902 (1999).

16 Wang, Z. B., Helander, M. G., Qiu, J., Liu, Z. W., Greiner, M. T. \& Lu, Z. H. Direct hole injection in to $4,4^{\prime}-\mathrm{N}, \mathrm{N}^{\prime}$-dicarbazole-biphenyl: a simple pathway to achieve efficient organic light emitting diodes. J. Appl. Phys. 108, 024510-024510 (2010).

17 Sun, K. \& Ouyang, J. Polymer solar cells using chlorinated indium tin oxide electrodes with high work function as the anode. Sol. Energy Mat. Sol. Cells 96, 238-243 (2012).

18 Zheng, S. \& Wong, K. Y. Enhanced performance in polymer solar cells by the use of a halogenated indium tin oxide anode. Appl. Phys. Lett. 102, 053302-053302 (2013).

19 Zhao, Y., Duan, L., Zhang, D., Qiao, J., Wang, L. \& Qiu, Y. Performance enhancement of organic light-emitting diodes by chlorinated indium tin oxide in the presence of hydrogen peroxide. Org. Electron 14, 882-887 (2013).

20 Xu, Z. Q., Li, J., Yang, J. P., Cheng, P. P., Zhao, J., Lee, S. T. \& Tang, J. X. Enhanced performance in polymer photovoltaic cells with chloroform treated indium tin oxide anode modification. Appl. Phys. Lett. 98, 253303 (2011).

21 Cao, X. A. \& Zhang, Y. Q. Performance enhancement of organic light-emitting diodes by chlorine plasma treatment of indium tin oxide. Appl. Phys. Lett. 100, 183304-183304 (2012).

22 Zhang, Y. Q., Acharya, R. \& Cao, X. A. Efficient and reliable green organic light-emitting diodes with $\mathrm{Cl}_{2}$ plasma-etched indium tin oxide anode. J. Appl. Phys. 112, 013103-013103 (2012)

23 Gao, C. H., Cai, S. D., Gu, W., Zhou, D. Y., Wang, Z. K. \& Liao, L. S. Enhanced hole injection in phosphorescent organic light-emitting diodes by thermally evaporating a thin indium trichloride layer. ACS Appl. Mat. Inter. 4, 5211-5216 (2012).

24 Kresse, G. \& Furthmüller, J. Efficiency of ab-initio total energy calculations for metals and semiconductors using a plane-wave basis set. Comput. Mater. Sci. 6, 15-50 (1996)

25 Kresse, G. \& Furthmüller, J. Efficient iterative schemes for ab initio total-energy calculations using a plane-wave basis set. Phys. Rev. B 54, 11169 (1996).

26 Blöchl, P. E. Projector augmented-wave method. Phys. Rev. B 50, 17953 (1994).

27 Kresse, G. \& Joubert, D. From ultrasoft pseudopotentials to the projector augmentedwave method. Phys. Rev. B 59, 1758 (1999).

28 Perdew, J. P., Burke, K. \& Ernzerhof, M. Generalized gradient approximation made simple. Phys. Rev. Lett. 77, 3865-3868 (1996).

29 Marezio, M. Refinement of the crystal structure of $\ln _{2} \mathrm{O}_{3}$ at two wavelengths. Acta Cryst. 20, 723-728 (1966).

30 Körber, C., Krishnakumar, V., Klein, A., Panaccione, G., Torelli, P., Walsh, A., Da Silva, J. L. F., Wei, S. -H., Egdell, R. G. \& Payne, D. J. Electronic structure of $\operatorname{In}_{2} \mathrm{O}_{3}$ and $\mathrm{Sn}$-doped $\mathrm{In}_{2} \mathrm{O}_{3}$ by hard $\mathrm{X}$-ray photoemission spectroscopy. Phys. Rev. B 81, 165207 (2010)

31 Milliron, D. J., Hill, I. G., Shen, C., Kahn, A. \& Schwartz, J. Surface oxidation activates indium tin oxide for hole injection. J. Appl. Phys. 87, 572-576 (2000).

32 Kim, K. P., Hussain, A. M., Hwang, D. K., Woo, S. H., Lyu, H. K., Baek, S. H., Jang, Y. \& Kim, J. H. Work function modification of indium-tin oxide by surface plasma treatments using different gases. Jpn J. Appl. Phys. 48, 1601 (2009).

33 Sun, X. H., Cheng, L. F., Liu, M. W., Liao, L. S., Wong, N. B., Lee, C. S. \& Lee, S. T. Photoelectron spectroscopic study of iodine-and bromine-treated indium tin oxides and their interfaces with organic films. Chem. Phys. Lett. 370, 425-430 (2003).

34 Helander, M. G., Greiner, M. T., Wang, Z. B., Tang, W. M. \& Lu, Z. H. Work function of fluorine doped tin oxide. J. Vac. Sci. Technol. A 29, 011019-011019 (2011).

35 Huheey, J. E., Keiter, E. A., Keiter, R. L. \& Medhi, O. K. Inorganic Chemistry: Principles of Structure and Reactivity. 4th Edn (Harper Collins, New York, 1993).

36 Henkelman, G., Arnaldsson, A. \& Jónsson, H. A fast and robust algorithm for Bader decomposition of charge density. Comput. Mater. Sci. 36, 354-360 (2006).

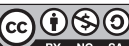

This work is licensed under a Creative Commons Attribution-NonCommercial-ShareAlike 3.0 Unported License. To view a copy of this license, visit http://creativecommons. org/licenses/by-nc-sa/3.0/ 